\title{
New Strategies in Enantioselective Hydrogenation on Supported Transition Metal Catalyst: Effects of Metal Sintering and Support Swelling
}

\author{
Nicolás R. Carrara, ${ }^{\circledR *, a, b}$ Juan M. Badano, ${ }^{a, c}$ Gustavo Mendow, ${ }^{a, c}$ Doris Ruiz, ${ }^{d}$ \\ Carlos R. Vera ${ }^{a, c}$ and Mónica E. Quiroga $a^{a, c}$ \\ anstituto de Investigaciones en Catálisis y Petroquímica, \\ Consejo Nacional de Investigaciones Científicas y Técnicas (INCAPE-CONICET), \\ Ruta 168, km 0, S3000AOJ-Santa Fe, Argentina \\ ${ }^{b}$ Facultad Regional Santa Fe, Universidad Tecnológica Nacional, Lavaise, 610, \\ S3004EWB-Santa Fe, Argentina \\ ${ }^{c}$ Facultad de Ingeniería Química, Universidad Nacional del Litoral (UNL), Santiago del Estero, \\ 2829, S3000AOJ-Santa Fe, Argentina \\ ${ }^{d}$ Departamento de Físico-Química, Facultad de Ciencias Químicas, Universidad de Concepción, \\ Casilla 160-C, Concepción, Chile
}

\begin{abstract}
The effect of noble metal catalysts supported on an ionic exchange resin for the enantioselective hydrogenation of ethyl pyruvate to ethyl lactate was studied. High activity and moderate enantioselectivity levels were obtained. Pt was the best enantioselective metallic phase, followed by $\mathrm{Pd}$ and $\mathrm{Au}$; $\mathrm{Rh}$ was the less enantioselective metallic phase. The effect of the active site was evaluated for the most enantioselective catalyst: 2Pt-WA30 (prefix indicates the metal loading). A novel method for sintering the Pt metal particles by nitriles reduction was used, and a controlled migration of the metal particles to the surface layers was observed. There existed an optimal particle size with maximization of the enantiomeric excess, corresponding to $24 \mathrm{~h}$ sintering. The hydrogenation of ethyl pyruvate to ethyl lactate was found to be structure-sensitive and the importance of the metal particle size for the adsorption of the chiral modifier was proved. Two different chirality induction methods were tried for the 2Pt-WA30 catalyst: $(i)$ the addition of the chiral modifier $(\mathrm{CM})$ to the reaction media, or (ii) the modification of the catalyst in a swelling medium prior to the reaction in order to encapsulate $\mathrm{CM}$. Lower activity but higher enantioselectivity levels were obtained with method (ii). Finally, the reuse of the catalysts was also assessed by means of consecutive reaction tests.
\end{abstract}

Keywords: ethyl pyruvate, chiral hydrogenation, sintering, modifier entrapment

\section{Introduction}

The synthesis of optically active drugs has gained importance in the last decades, mainly because of the possible adverse effects of the use of the wrong optical isomers. ${ }^{1}$ In terms of atomic economy, asymmetric catalysis has become the most successful technique because it uses one step-reactions to afford optically active compounds from available reagents and catalysts. ${ }^{2}$ Asymmetric hydrogenation reactions are powerful tools for the production of chiral compounds. ${ }^{3}$ Catalytic heterogeneous hydrogenation can be performed using a chiral modifier

*e-mail: ncarrara@ fiq.unl.edu.ar
(CM) for the modification of catalytic surfaces. ${ }^{4}$ The Pt-cinchonidine system for the enantioselective hydrogenation of $\alpha$-keto-esters is a successful example of catalytic system. It was first reported by Orito ${ }^{5}$ using high Pt loadings and several complex preparation and activation procedures. Excellent enantio differentiation values up to $90-95 \%$ were achieved with this system, ${ }^{6}$ the heterogeneous catalyst is recovered, but as CM is dissolved in the same phase of the reactants and products, the liquid phase, its separation at the end of the reaction was difficult, too expensive and environmental not friendly. The recyclability of CM seems to be a serious problem, not only from an economic viewpoint, but more precisely because of environmental issues. ${ }^{2}$ 
It has been demonstrated that chiral hydrogenation of ethyl pyruvate is a structure-sensitive reaction. ${ }^{7}$ Thermal treatments could be applied on catalysts for sintering the metal particles. Attard et al. ${ }^{8}$ applied thermal sintering to $5 \% \mathrm{Pt} / \mathrm{C}$ in a reducing atmosphere. They found that the crystallinity of the particles was improved as increased the treatment temperature and that an optimal enantioselectivity was achieved for a metal particle size of $8 \mathrm{~nm}$, corresponding to a $700 \mathrm{~K}$ thermal treatment. ${ }^{8}$

Ionic exchange resins have been used as supports for several applications, including hydrogenation of different substrates. ${ }^{9}$ Resins have an open pore structure and pose no mass transfer problems to reactants and products. They have negligible solubility in most solvents and enable an easy preparation of catalysts..$^{10}$ Moreover, the open structure and low metal-support interaction could favor the metal cluster mobility and reduce the active phase dispersion. ${ }^{11}$ Another interesting property is known as swelling. During swelling, resins can increase its volume in a polar solvent up to $800 \%$ depending on the crosslinking percentage. ${ }^{9}$ This property can be used for the catalyst modification before the reaction, with the aim of a more effective modification or for trapping CM inside the catalyst structure. To our knowledge, only a few publications using resins as supports for enantioselective hydrogenation are available. Moreover, no publication of these materials taking advantage of these properties and applied in asymmetric catalysis is available.

In this work, different catalysts based on noble metals $(\mathrm{Pt}, \mathrm{Pd}, \mathrm{Rh}$ and $\mathrm{Au})$ supported over an ionic exchange resin were prepared. The effect of the metallic active site on the activity and enantioselectivity was evaluated during the hydrogenation of ethyl pyruvate (EP) to $(R)$ - or $(S)$ ethyl lactate $((R)-/(S)$-EL) in order to evaluate the activity, enantioselectivity and determine the best active phase. After the metal screening step, a further analysis was performed over the best catalyst and the effects of metal particle size and chirality of the modifier structure ((-)-cinchonidine $(\mathrm{CD})$ or $(+)$-cinchonine $(\mathrm{CN})$ ) were thus evaluated. As thermal treatments on resins are temperature limited (maximum temperature $393 \mathrm{~K}$ ) and not suitable for these catalysts, another method for metal sintering was tested that is based on the work by Mendow, ${ }^{11}$ who observed metal sintering of $2 \% \mathrm{Pd} / \mathrm{WA} 30$ catalyst during the aqueous phase reduction of nitrites.

The use of Orito's method ${ }^{5}$ for catalyst modification was compared to a new method involving swelling of the support. This novel method involved swelling of the catalyst support in a solvent containing the chiral modifier $(\mathrm{CM})$, in order to trap the latter in the swelled pores.
The effectiveness of this modification on activity and enantioselectivity was analyzed and the reuse of catalysts was also evaluated.

\section{Experimental}

\section{Catalyst preparation}

A Mitsubishi WA30 anionic exchange resin was used as support. All the catalysts were prepared by ionic exchange using the following procedure: $10 \mathrm{~g}$ of WA30 support were immersed in a $30 \mathrm{~mL}$ aqueous solution of metal precursor $\left(\mathrm{H}_{2} \mathrm{PtCl}_{6}, \mathrm{PdCl}_{2}, \mathrm{AuCl}_{3}\right.$ and $\left.\mathrm{RhCl}_{3}\right)$ at $\mathrm{pH} 1$ (adjusted with $1 \mathrm{M} \mathrm{HCl}$ ). Volume and concentration of the impregnating solution were adjusted to yield the desired metal concentration on the final catalysts: 1 wt.\% of $\mathrm{Au}$ and Pt, and 2 wt.\% of Pt, Pd and Rh. The solution concentration used were: $0.0169 \mathrm{M}$ for $\mathrm{AuCl}_{3}$, $0.0017 \mathrm{M}$ or $0.0034 \mathrm{M}$ for $\mathrm{H}_{2} \mathrm{PtCl}_{6}, 0.0626 \mathrm{M}$ for $\mathrm{PdCl}_{2}$ and $0.0648 \mathrm{M}_{\text {for }} \mathrm{RhCl}_{3}$. Impregnation lasted $3 \mathrm{~h}$ and was performed at $298 \mathrm{~K}$, under nitrogen blanketing and mild stirring $(120 \mathrm{rpm})$. Then the samples were filtered and dried at $353 \mathrm{~K}$ for $24 \mathrm{~h}$. The catalyst samples were named: 1Au-WA30, 1Pt-WA30, 2Pt-WA30, 2Pd-WA30 and $2 \mathrm{Rh}-\mathrm{WA} 30$, the prefix indicates the metal loading. Hydrazine was used as reducing agent in 1:5 (metalreducer) molar ratio, at $298 \mathrm{~K}$ and $\mathrm{pH} 10$ (adjusted with $1 \mathrm{M} \mathrm{NaOH}$ ) for $2 \mathrm{~h}$. Finally, the samples were filtered and dried at $353 \mathrm{~K}$ for $24 \mathrm{~h}$.

On the other hand, in order to study the effect of metallic particle size over the most enantioselectivity system, $5 \mathrm{~g}$ of 2Pt-WA30 catalyst were sintered by the nitrite reduction reaction in a semi-continuous glass reactor. The liquid phase was an aqueous solution of $100 \mathrm{~mL}$ containing 10,000 ppm of $\mathrm{KNO}_{2}$. Hydrogen was bubbled at $20 \mathrm{~mL} \mathrm{~min}{ }^{-1}$, at a pressure of $1 \mathrm{bar}$, and the solution was gently stirred at $300 \mathrm{rpm}$ and room temperature. ${ }^{12}$ Samples of the sintered catalysts $(0.5 \mathrm{~g})$ were taken at sintering times $(\Phi)$ of 6,12 , 24 or $48 \mathrm{~h}$. Samples were named as 2Pt-WA30- $\Phi$.

Besides, in order to evaluate the modification technique on the catalyst surface prior to the reaction, a swelling technique was used on the reduced 2Pt-WA30 catalyst. The catalyst sample ( $\mathrm{W}_{\text {cat }}=0.25 \mathrm{~g}$, catalysts mass $)$ was exposed with moderate stirring (300 rpm) to a solution of (-)-inchonidine/methanol (volume of $60 \mathrm{~mL}, \mathrm{Cd}$ concentration of $0.02 \mathrm{~g} \mathrm{~L}^{-1}$ ) at room temperature for $2 \mathrm{~h}$. Then, it was washed with cyclohexane and dried at $353 \mathrm{~K}$ for $12 \mathrm{~h}$, and named 2Pt-WA30-SW.

Before catalytic tests, all the samples were pretreated with $\mathrm{H}_{2}$ flow $\left(20 \mathrm{~mL} \mathrm{~min}^{-1}\right)$ at $393 \mathrm{~K}$ for $60 \mathrm{~min}$. 


\section{Characterization tests}

The bulk metal composition of the catalysts was assessed by X-ray fluorescence (XRF) with a Shimadzu EDX-720 equipment. Prior to the analysis, samples were dried in an oven at $353 \mathrm{~K}$, and then, the measures were performed under vacuum.

The catalysts were further characterized by nitrogen sortometry on a Quantachrome equipment. Samples were pre-treated under vacuum at $373 \mathrm{~K}$ for $1 \mathrm{~h}$. The nitrogen adsorption-desorption isotherms were obtained with relative pressures $\left(\mathrm{p} / \mathrm{p}_{\mathrm{o}}\right)$ between 0.05 and 0.988 , and the Brunauer-Emmett-Teller (BET) model was used to calculate the specific surface area.

Optical micrographs were obtained with a Mitsubishi Microwatcher VS-30H Microscope and a Sony color video printer. X-ray diffraction (XRD) was performed by a Shimadzu XD-1 equipment, using $\mathrm{Cu} \mathrm{K} \alpha$ radiation $(\lambda=1.5405 \AA)$ filtered with $\mathrm{Ni}$ and a scan speed of 1 degree $\mathrm{min}^{-1}$. Thermogravimetric analysis (TGA) was carried out using a TA Instruments 2950 apparatus. The samples (ca. $10 \mathrm{mg}$ ) were heated in a flow of air or $\mathrm{N}_{2}$ $\left(40 \mathrm{~mL} \mathrm{~min}^{-1}\right)$ from room temperature up to $900 \mathrm{~K}$ at a heating rate of $5 \mathrm{~K} \mathrm{~min}^{-1}$.

Transmission electron microscopy (TEM) was performed in a JEOL $100 \mathrm{CX}$ Transmission Electron Microscope, operated at $100 \mathrm{kV}$. The samples were prepared by grinding the pellets, suspending in ethanol and sonicating for $15 \mathrm{~min}$. After that, a drop of this suspension was placed on a 200 mesh copper grid with a Formvar ${ }^{\circledR}$ film to be observed in the equipment. For each sample, a set of digital images were taken in order to make the phases identification and measure the particle diameters to obtain the particle size distributions, the Digital Micrograph software was used.

X-ray photoelectron spectroscopy (XPS) measurements were carried out with a Multitech UniSpecs equipment following procedures previously reported. ${ }^{13-15}$ Prior to the analysis the samples were reduced in $\mathrm{H}_{2}$ flow at $393 \mathrm{~K}$.

\section{Catalytic tests}

The enantioselective hydrogenation of ethyl pyruvate (EP) was carried out in a $60 \mathrm{~mL}$ batch polytetrafluorethylene (PTFE) coated stainless steel tank reactor, with a mechanical stirrer. The reaction conditions were: $\mathrm{W}_{\text {cat }}=0.25 \mathrm{~g}$ catalysts mass, $0.176 \mathrm{M}$ initial concentration of ethyl pyruvate $\left(\mathrm{C}_{\mathrm{EP}}^{\circ}\right)$, 0.01-0.034 $\mathrm{g} \mathrm{L}^{-1}$ initial concentration of chiral modifier $\left(\mathrm{C}^{\circ}{ }_{\mathrm{CM}}\right)$, temperature at $298 \mathrm{~K}$ and 40 bar of hydrogen pressure, liquid phase volume of $50 \mathrm{~mL}$. The solvent for the reaction was cyclohexane ( $\varepsilon=2.02$, dielectric constant). It has been proved that apolar solvents ( $\varepsilon$ in the range 2-10) favor the open 3 conformation of the alkaloid which is the necessary to achieve high enantioselectivity. ${ }^{16,17}$ The tests were carried out in triplicates, within an experimental error of $3 \%$. Reactant and products were analyzed by gas chromatography with flame ionization detection (GC-FID) using a $30 \mathrm{~m}$ Supelco $\beta$-DEX 110 capillary column. The enantiomeric excess (ee) was calculated as

ee $(\%)=\frac{\left(\mathrm{Y}_{(\mathrm{R})}-\mathrm{Y}_{(\mathrm{S})}\right)}{\left(\mathrm{Y}_{(\mathrm{R})}+\mathrm{Y}_{(\mathrm{S})}\right)} 100 \%$

where Y means yield and the subscript correspond to the $R$-EL and $S$-EL isomers of the Prelog-Ingold-Cahn notation.

\section{Results and Discussion}

\section{Catalyst characterization results}

Metal loadings of the samples were verified by XRF, and the experimental metal concentration values were similar to the nominal theoretical ones to an error of about $5 \%$.

Nitrogen physisorption tests were performed on the support, 2Pt-WA30 and 2Pt-WA30-24, respectively fresh and the $24 \mathrm{~h}$ sintered catalyst. Figure 1 presents the isotherms of: (a) the fresh and (b) $24 \mathrm{~h}$ sintered samples, and (c) pore volume loss between fresh and sintered catalysts. The obtained isotherms are of type II, indicating the presence of an open pore structure and the absence of hysteresis phenomena. The pore distribution was obtained by means of the Barrett-Joyner-Halenda method. The estimated specific surface area for WA30 support, 2Pt-WA30 fresh and $24 \mathrm{~h}$ sintered samples were 18.5, 17.4 and $17.2 \mathrm{~m}^{2} \mathrm{~g}^{-1}$, respectively. A comparison of the freshly prepared resin supported catalyst and the sintered one indicates that about $34 \%$ of the pore volume was lost upon sintering of the metal phase and that this volume was concentrated in the 3-11 nm diameter range, corresponding to mainly meso- and macropores. ${ }^{18}$ This relative large pore volume loss must be due to the preferential concentration of the metal particles in pore mouths.

Figure 2 presents optical micrographs (OM) of support and catalyst fragments. The pellets have a spherical shape. The observed pellet diameters were in the range 0.3-1.1 mm.

Results of XRD are shown in Figure 3. As the resin was amorphous, no signals of the support were detected. Peaks attributed to the reflections of noble metal crystalline planes 

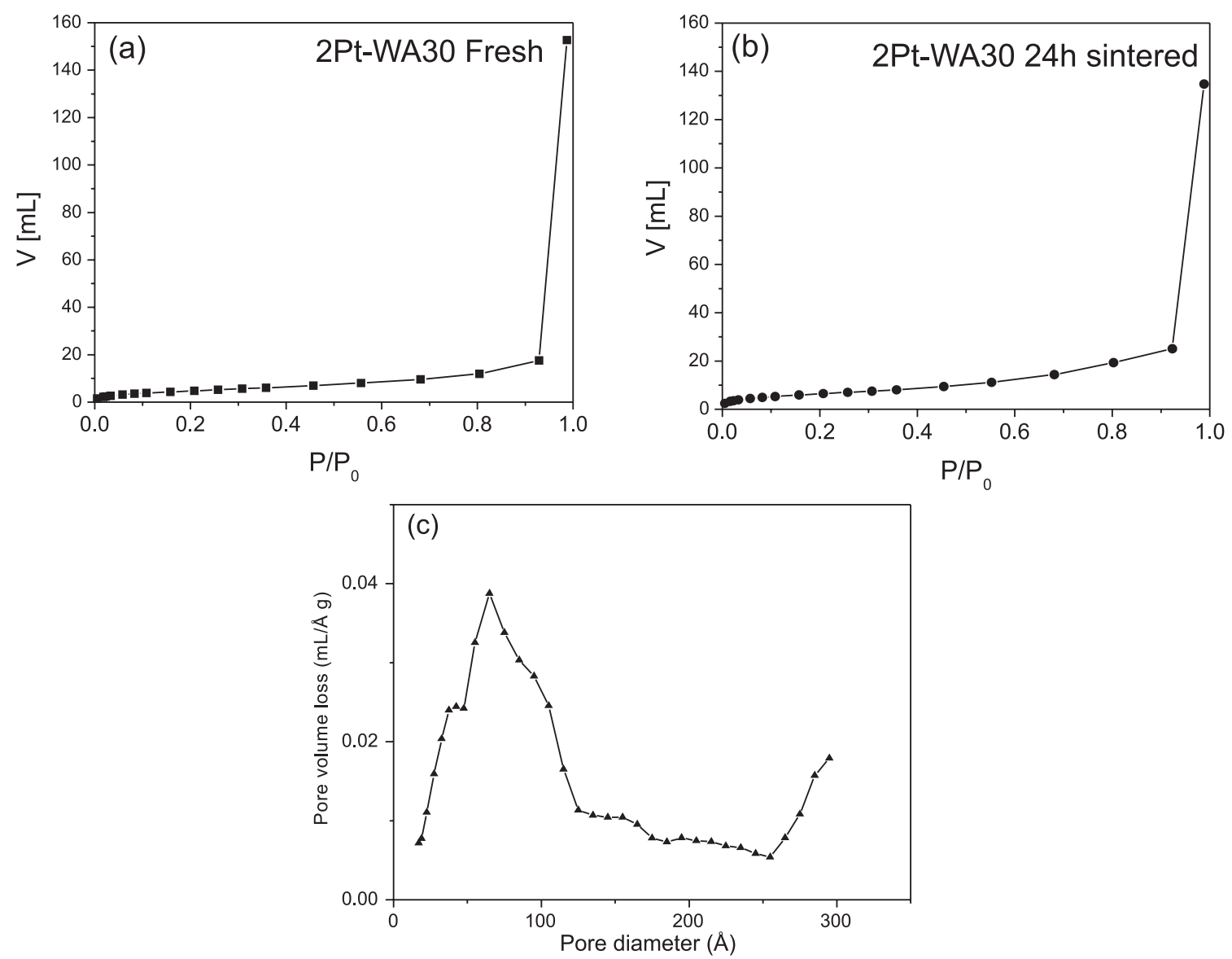

Figure 1. Adsorption isoterms of (a) fresh and (b) $24 \mathrm{~h}$ sintered 2Pt-WA30 catalyst, and (c) pore volume loss between fresh and sintered sample.

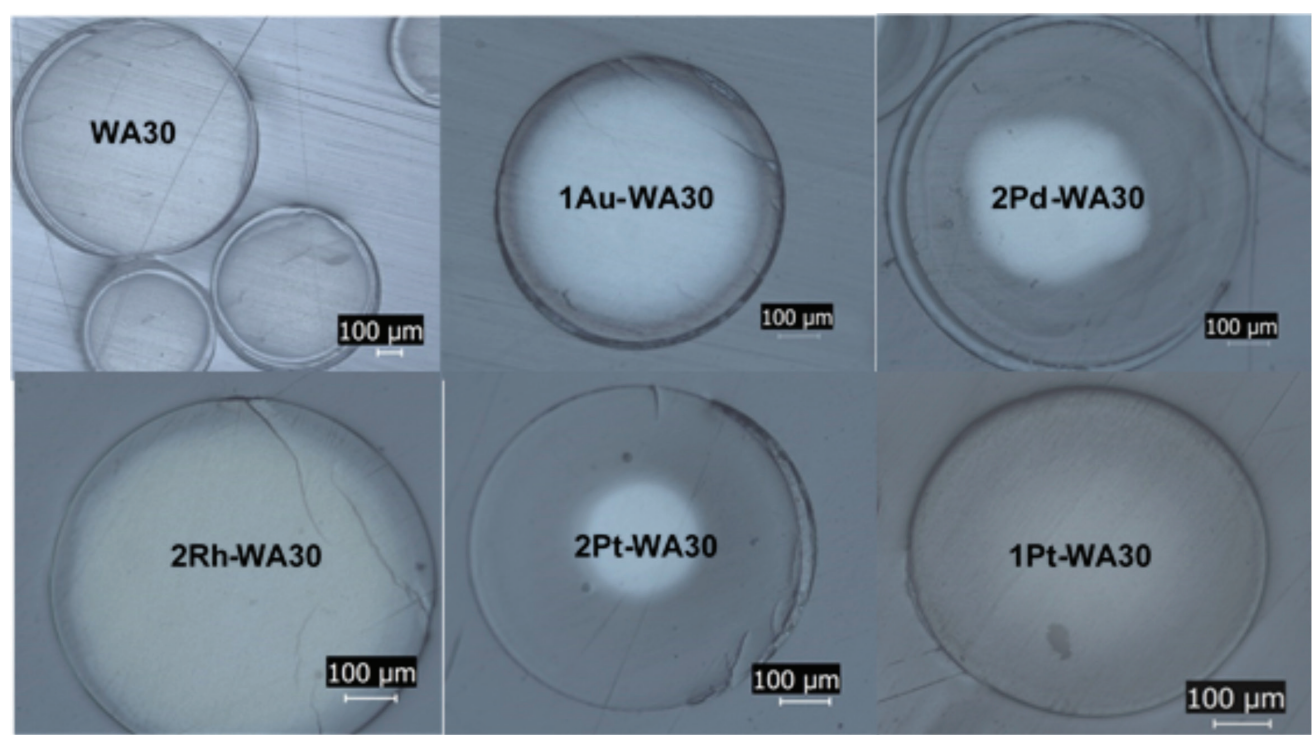

Figure 2. Optical micrographs of support and catalyst fragments.

were found in the diffractograms of fresh and sintered samples: $\operatorname{Pd}(111)$ at $39.9^{\circ}, \operatorname{Pd}(200)$ at $46.0^{\circ}, \operatorname{Rh}(111)$ at $41.2^{\circ}, \mathrm{Rh}(200)$ at $47.9^{\circ}, \mathrm{Au}(111)$ at $38.1^{\circ}, \mathrm{Au}(200)$ at $44.3^{\circ}$, $\mathrm{Pt}(111)$ at $40.0^{\circ}$ and $\mathrm{Pt}(200)$ at $46.6^{\circ} . .^{19-24}$ The Pt-WA30- $\Phi$ sintered catalysts, if compared to the untreated ones, maintained identical proportion of the $\mathrm{Pt}(111)$ and $\mathrm{Pt}(200)$ crystalline planes.

In Figure 4, thermal gravimetric tests are plotted, showing the stability of the resin and Pt catalysts. The obtained results allow selecting the appropriate 


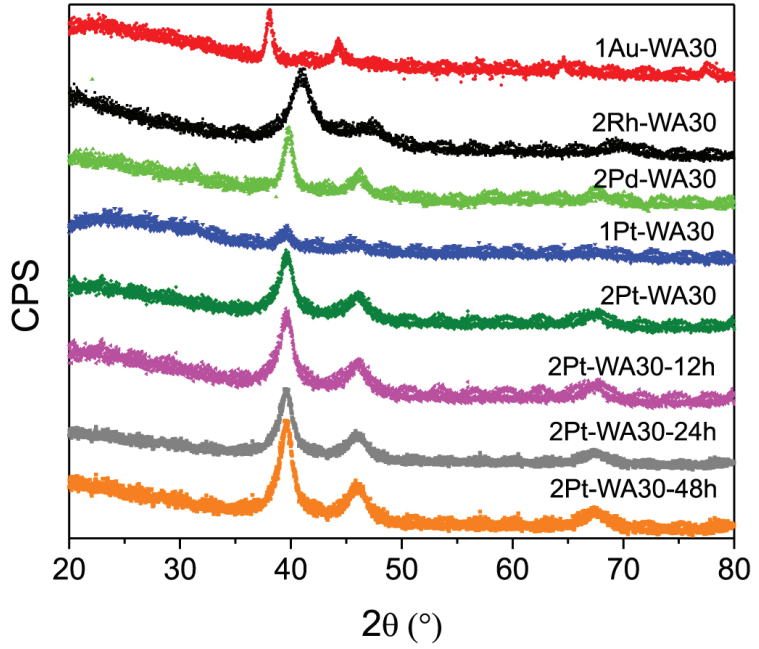

Figure 3. XRD diffractograms of the fresh M-WA30 and Pt-WA30- $\Phi$ sintered catalysts.

temperatures for thermal pretreatment and reaction. The thermal degradation patterns of the WA30 support in the presence of oxidant (air) or inert atmosphere $\left(\mathrm{N}_{2}\right)$ can be compared. It can be seen that these materials begin to lose weight noticeably at about $523 \mathrm{~K}$. At high temperatures, a rapid decomposition of the organic phase of the support occurs. At the end of the test, a residue remains of ca. 30 or 5\% the initial mass, respectively for inert or oxidant atmosphere. The decomposition of the organic matrix in the 523-700 K range would correspond to the consequence of cracking, depolimerization, decomposition and oxidation reactions. ${ }^{25}$ The catalysts are slightly more unstable than the support due to the catalytic action of the metal. ${ }^{13}$

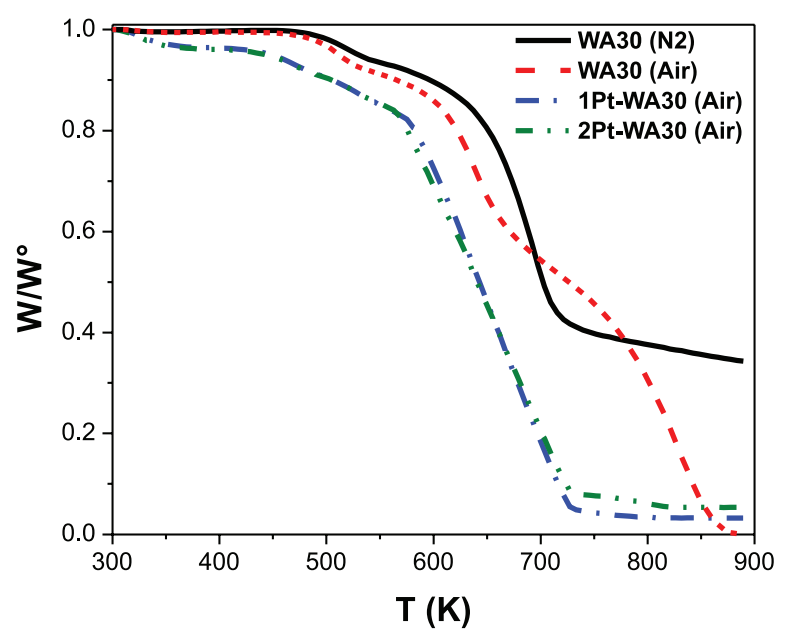

Figure 4. Thermogravimetry results of support and Pt catalysts in $\mathrm{N}_{2}$ or air atmosphere.

Electronic micrographs of the support and Pt catalysts can be seen in Figure 5. No metallic phase is observed in the micrograph of the support. After the ionic exchange, the metallic phase on the 2Pt-WA30 sample can be distinguished as dark points. After the sintering treatment, an agglomeration of metal particles was observed, and the size of the particles increased with the sintering time, as

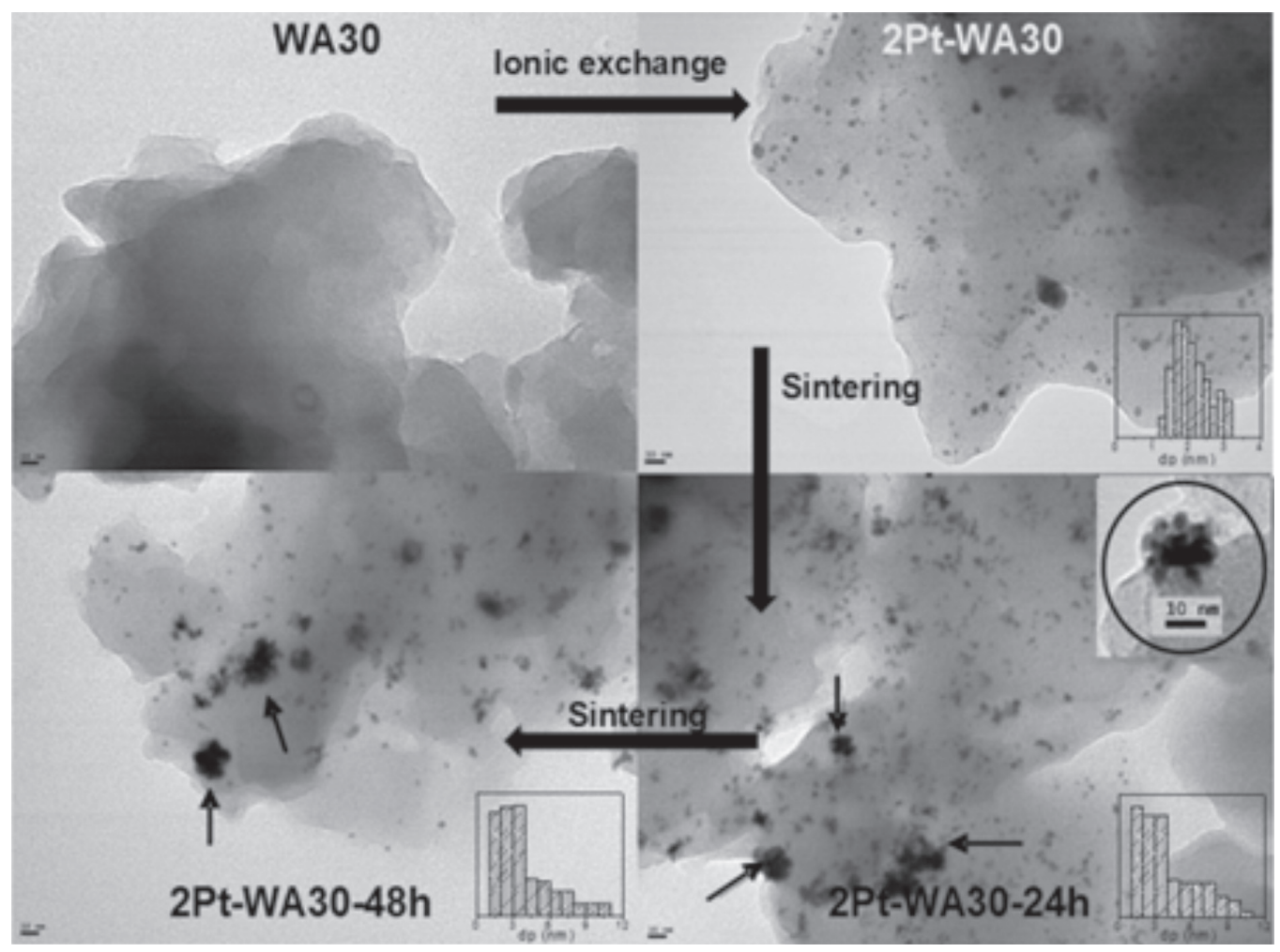

Figure 5. TEM images of support and Pt catalyst fragments. 
seen in the 2Pt-WA30-24h and 2Pt-WA30-48h samples. It was observed by XRD that the growing crystals maintained crystallinity and faceted particles were formed.

Metal particle size distribution was obtained from TEM analysis. Values of the average volume-surface diameter $\left(\mathrm{d}_{\mathrm{TEM}}\right)$ were calculated as follows:

$\mathrm{d}_{\mathrm{TEM}}=\frac{\sum \mathrm{n}_{\mathrm{i}} \mathrm{d}_{\mathrm{i}}^{3}}{\sum \mathrm{n}_{\mathrm{i}} \mathrm{d}_{\mathrm{i}}^{2}}$

Taking this $d_{\text {TEM }}$ values for each catalyst, the metal dispersion $\left(\mathrm{D}_{\text {TEM }}\right)$ of the catalysts was estimated following a procedure explained by Badano et al..$^{26}$ The values of $\mathrm{d}_{\text {TEM }}$ and the calculated $\mathrm{D}_{\text {TEM }}$ are informed in Tables 1 (fresh catalysts) and 2 (sintered 2Pt-WA30- $\Phi$ catalyst). As it can be seen in Table 1, the 2Rh-WA30 catalyst has the best metal dispersion with a value of $75.7 \%$, followed by $\mathrm{Pt}$ and Pd catalysts with values between 52.6 and $44.8 \%$, respectively. The less dispersed sample was $1 \mathrm{Au}-\mathrm{WA} 30$ with a value of $19.5 \%$. The sintered catalysts had smaller metal dispersion due to the increase of the average $\mathrm{d}_{\mathrm{TEM}}$ values. The metal sintering in the 2Pt-WA30 catalyst was favored by the low metal-support interaction over the weak anionic exchange resin, and the volume increase by swelling in aqueous media. ${ }^{27}$ Loss in metallic surface area was compensated by the formation of more suitable metallic sites for alkaloid adsorption.

Values of binding energy, $\mathrm{Pd}$ and $\mathrm{Rh} 3 \mathrm{~d}_{5 / 2}, \mathrm{Au}$ and $\mathrm{Pt} 4 \mathrm{f}_{7 / 2}$ and chloride/metal $(\mathrm{Cl} / \mathrm{Me})$ atomic ratios can be found in Table 1. A peak at ca. $198.5 \mathrm{eV}$ corresponding to chloride (not eliminated during the pretreatments) was detected for all the samples. Pt catalysts showed the lowest $\mathrm{Cl} / \mathrm{Me}$ atomic ratio values, while the other catalysts showed higher values. $\mathrm{BE}$ values suggested two kinds of species on the surface: $\mathrm{Me}^{\circ}$ and $\mathrm{Me}^{\delta+}$, with $\delta \leq 3$ for $\mathrm{Rh}, \delta \leq 2$ for $\mathrm{Pd}$ or $\mathrm{Pt}$ and $\delta \leq 1$ for $\mathrm{Au}^{28} \mathrm{Me}^{\delta+}$ electron deficient species were possibly linked to the presence of non-reduced $\mathrm{Me}$ species stabilized by neighbouring $\mathrm{Cl}$ atoms. ${ }^{26} \mathrm{In}$ all cases, the metallic species was in higher proportion, indicating that the reduction procedure was effective.

\section{Ethyl pyruvate hydrogenation results}

First of all, two blank tests were performed: (i) a reaction in absence of catalyst and (ii) a reaction with the WA30 resin support, without the metallic phase. In both cases, no activity was observed. Also, a preliminary experiment of EP hydrogenation over the 2Pt-WA30 catalyst in the absence of $\mathrm{CM}$ was made; no enantiomeric excess of EL was detected and a diminution of the reaction rate was observed, in agreement to results obtained by other authors. ${ }^{29,30}$ Then, a screening of the metallic active phase was made using the set of 1Au-WA30, 1Pt-WA30, 2Pt-WA30, 2Pd-WA30 and 2Rh-A30. In all cases, CD was used as chiral inducer, leading to the preferential formation of $(R)$-EL. The results of EP total conversion $\left(\mathrm{X}_{\mathrm{EP}}\right)$ and $(R)$-EL enantioselectivity (ee) are shown in Table 2 and in Figure 6. Initial concentration of ethylpyruvate and cinchonidine are indicated as $[\mathrm{EP}]$ and $[\mathrm{CD}]$, respectively.

In all cases, the reaction was maintained until total conversion was achieved. It can be seen that all catalysts were active for the hydrogenation of ethyl pyruvate to ethyl lactate. The order of activity was: 2 Rh-WA30 >> 1Pt-WA30 $>$ 2Pt-WA30 > 2Pd-WA30 > 1Au-WA30. The performance of the catalysts can be interpreted on the one hand, in terms of electronic effects related to the high electron deficiency of $\mathrm{Me}^{\delta+}$ (Lewis acidic site) that attracts electrons from the oxygen (Lewis basic site) of the carboxylic group of EP, favoring its adsorption. $\mathrm{Rh}^{3+}$ sites would favor the EP surface adsorption more easily than $\mathrm{P}^{2+}$ or $\mathrm{Pd}^{2+}$, otherwise, the adsorption of the substrate should be less likely on $\mathrm{Au}^{+}$with low attraction to EP. Besides, $\mathrm{Me}^{\delta+}$ sites could favor the adsorption of the chiral modifier. On the other hand, $\mathrm{Me}^{\circ}$ sites, metallic centres rich in electrons, can cleave the bonds in $\mathrm{H}_{2}$ by means of the interaction of a filled d metal orbital with the empty sigma antibonding

Table 1. Values of metal dispersion $\left(\mathrm{D}_{\mathrm{TEM}}\right)$, metal particle diameter size $\left(\mathrm{d}_{\mathrm{TEM}}\right)$, XPS binding energy (BE) (atomic ratios in parenthesis) and $\mathrm{Cl} / \mathrm{Me}$ atomic ratio

\begin{tabular}{|c|c|c|c|c|c|}
\hline \multirow{3}{*}{ Catalyst } & \multirow{3}{*}{$\mathrm{D}_{\mathrm{TEM}} / \%$} & \multirow{3}{*}{$\mathrm{d}_{\mathrm{TEM}} / \mathrm{nm}$} & \multicolumn{3}{|c|}{ X-ray photolectron spectrosocpy (XPS) data ${ }^{a}$} \\
\hline & & & \multicolumn{2}{|c|}{$\mathrm{BE} / \mathrm{eV}$} & \multirow{2}{*}{$\mathrm{Cl} / \mathrm{Me}$} \\
\hline & & & $\mathrm{Me}^{\mathrm{o}}$ & $\mathrm{Me}^{\delta+}$ & \\
\hline 1Au-WA30 & 19.5 & 7.3 & $84.3(94.3 \%)$ & $86.3(5.7 \%)$ & 0.3002 \\
\hline 1Pt-WA30 & 52.6 & 2.1 & $71.6(81.9 \%)$ & $73.6(18.1 \%)$ & 0.0628 \\
\hline 2Pt-WA30 & 49.2 & 2.3 & $71.5(85.3 \%)$ & $73.9(14.7 \%)$ & 0.0250 \\
\hline 2Pd-WA30 & 44.8 & 2.5 & $336.4(87.4 \%)$ & $338.7(12.6 \%)$ & 0.2489 \\
\hline 2Rh-WA30 & 75.7 & 1.4 & $307.8(77.7 \%)$ & $310.2(22.3 \%)$ & 0.1595 \\
\hline
\end{tabular}

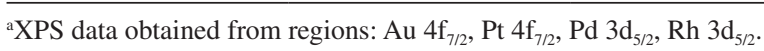


Table 2. Ethyl pyruvate (EP) total conversion $\left(\mathrm{X}_{\mathrm{EP}}\right),(R)$-EL enantioselectivity $\left(\mathrm{ee}_{(\mathrm{R})}\right)$, initial reaction rate of EP $\left(\mathrm{r}^{\circ}\right)$ and external $\mathrm{d}$ orbital configuration of the characterized metal species $\left(\mathrm{d}^{\mathrm{n}}\right)$

\begin{tabular}{|c|c|c|c|c|c|}
\hline \multirow{2}{*}{ Catalyst } & \multirow{2}{*}{$\begin{array}{l}X_{E P} / \% \\
t=20 h\end{array}$} & \multirow{2}{*}{$\begin{array}{l}\mathrm{ee}_{(\mathrm{R})} / \% \\
\mathrm{t}=20 \mathrm{~h}\end{array}$} & \multirow{2}{*}{$\begin{array}{c}\mathrm{r}^{\circ} \times 10^{3} / \\
\left(\mathrm{mol} \mathrm{g}_{\mathrm{Me}}{ }^{-1} \mathrm{~min}^{-1}\right)\end{array}$} & \multicolumn{2}{|c|}{ Metal external d $\mathrm{d}^{\mathrm{n}}$ orbital } \\
\hline & & & & $\mathrm{Me}^{\circ}$ & $\mathrm{Me}^{+}$ \\
\hline 1Au-WA30 & 37 & 15.6 & 4.576 & $5 d^{10}$ & $5 d^{10}$ \\
\hline 1Pt-WA30 & 64 & 19.6 & 7.744 & $5 d^{9}$ & $5 \mathrm{~d}^{\mathrm{n}}(8 \leq \mathrm{n}<9)$ \\
\hline 2Pt-WA30 & 53 & 21.6 & 2.640 & $5 d^{9}$ & $5 \mathrm{~d}^{\mathrm{m}}(8 \leq \mathrm{n}<\mathrm{m}<9)$ \\
\hline 2Pd-WA30 & 42 & 16.5 & 3.168 & $4 d^{10}$ & $4 \mathrm{~d}^{\mathrm{n}}(8 \leq \mathrm{n}<10)$ \\
\hline 2Rh-WA30 & 90 & 8.2 & 4.224 & $4 d^{8}$ & $4 \mathrm{~d}^{\mathrm{n}}(6 \leq \mathrm{n}<8)$ \\
\hline
\end{tabular}

t: time; reaction conditions: $\mathrm{W}_{\text {cat }}=0.25 \mathrm{~g}, \mathrm{~V}_{\text {solvent }}=50 \mathrm{~mL},[\mathrm{EP}]=0.176 \mathrm{M},[\mathrm{CD}]=0.034 \mathrm{~g} \mathrm{~L}^{-1}, \mathrm{~T}=298 \mathrm{~K}, \mathrm{p}_{\mathrm{H} 2}=40$ bar.

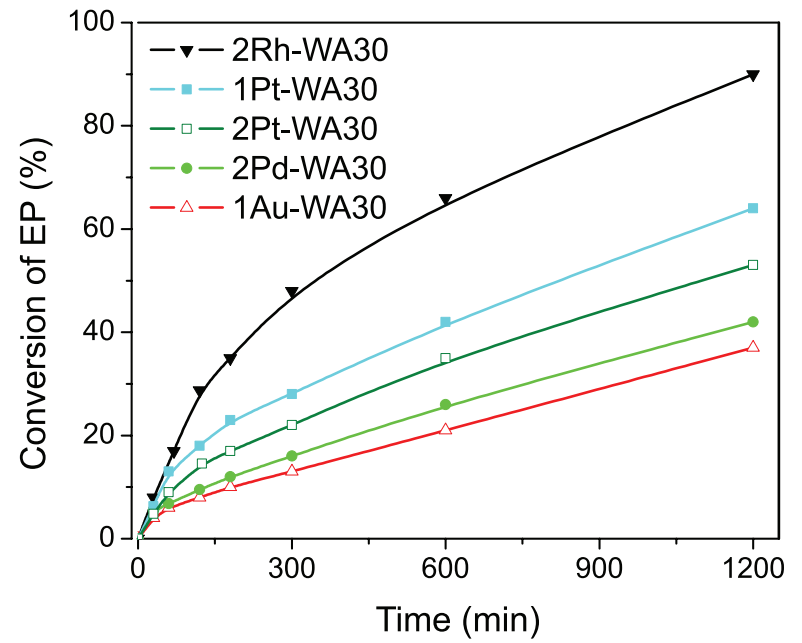

Figure 6. EP Conversion as function of reaction time. 2Rh-WA30 $(\boldsymbol{\nabla})$, 1Pt-WA30 (ם), 2Pt-WA30 $(\bullet), 2 P d-W A 30(\triangle)$ and 1Au-WA30 $(\Delta)$. Conditions: $\mathrm{W}_{\text {cat }}=0.25 \mathrm{~g}, \mathrm{~V}_{\text {solvent }}=50 \mathrm{~mL},[\mathrm{EP}]=0.176 \mathrm{M}$, $[C D]=0.034 \mathrm{~g} \mathrm{~L}^{-1}, \mathrm{~T}=298 \mathrm{~K}, \mathrm{p}_{\mathrm{H} 2}=40$ bar.

molecular orbital of $\mathrm{H}_{2} \cdot{ }^{31}$ The rupture of the hydrogen bond is more easily done on metals with a high concentration of available electrons in the external $\mathrm{d}$ orbital, ${ }^{31}$ as it is the case of palladium or gold (Table 2). As for the active sites used, the rate limiting step is the adsorption of the substrate, ${ }^{14}$ so the high activity of $2 \mathrm{Rh}-\mathrm{WA} 30$ could be related to $\mathrm{Rh}^{3+}$ sites which favor the EP surface adsorption. While the low activity of $1 \mathrm{Au}-\mathrm{WA} 30$ catalyst could be related to low charge of $\mathrm{Au}^{+}$. Therefore, the differences in activity between the reported catalysts could be partly attributed to differences in adsorption strength and electronic effects due to $\mathrm{Me}^{\delta+}$ and to the electronic density of the external $\mathrm{d}^{\mathrm{n}}$ orbital of each metal. Besides, effects of the average particle diameter of the catalysts could not be discarded.

As observed in Table 2, the order of enantioselectivity was: 2 Pt-WA30 $>1$ Pt-WA30 $>2$ Pd-WA30 $>1$ Au-WA30 $>>$ 2Rh-WA30. In terms of enantioselectivity, Pt catalysts were informed in the literature as the best metal phase to be chirally modified for the enantioselective hydrogenation of $\alpha$-ketoesters. That capacity could be attributed to the good interaction of the active metal site with the chiral modifier. ${ }^{1}$ Other metal phases studied in the literature showing a poorer enantiodifferentiation capacity during ethyl pyruvate hydrogenation were Ir, Pd, Rh, Ru and Ni. In our work, the metal screening experiments were performed with a high concentration of $\mathrm{CM}$ cinchonidine to minimize the presence of unmodified sites. The presence of the chiral modifier can improve the reaction rate up to 20-30 times, as observed by several authors. ${ }^{2,32,33}$ The high coverage of $\mathrm{CM}$ on the catalyst surface may affect the activity of the less dispersed catalysts because they have affinity for the metal sites and avoid $\mathrm{H}_{2}$ chemisorption, especially in the case of the Au catalyst, the one with the biggest particle size. The lowest enantiomeric excess was obtained with the 2Rh-WA30 catalyst, which has the smallest particle size. Better results were achieved with the 2Pd-WA30 and 1Au-WA30 catalysts, 16.5 and $15.6 \% \mathrm{ee}_{(\mathrm{R})}$, respectively. The best results were obtained with the Pt catalysts: 1Pt-WA30 and 2Pt-WA30 catalysts with ee ${ }_{(\mathrm{R})}$ values of 19.6 and $21.6 \%$, respectively. This trend was in agreement with other reports in the scientific literature. ${ }^{1,30,32}$ The higher enantiomeric excess achieved with the 2Pt-WA30 catalyst was attributed to the bigger metal particle size of 2Pt-WA30 in comparison to 1Pt-WA30 (2.3 and $2.1 \mathrm{~nm}$, respectively). No significant differences in BE of the XPS spectra of both samples were found.

The asymmetric hydrogenation of ethyl pyruvate is a structure-sensitive reaction. An adequate particle size improves the metal-CM interaction, increases the amount of chiral sites and enhances the enantioselectivity. According to the literature, a particle diameter between $4-10 \mathrm{~nm}$ is considered to be optimal. ${ }^{1}$ In this sense, different catalyst synthesis routes and conditioning treatments could be used to control the metal particle size.

Due to the good performance of 2Pt-WA30, this catalyst was selected for further studies of sintering and swelling. The effect of metal particle size and the effect of encapsulation of the chiral modifier inside the resin 
pores by swelling had to be studied in more detail. Over resin supports sintering is favored by the low metal-support interaction, especially over weak anionic exchange resins. Sintering thermal treatments are known to lead to the formation of metal particles with a high concentration of low Miller index planes. Surface sites on these planes are highly coordinated and are favorable for the adsorption of chiral modifiers, thus leading to material with high enantiomeric activity. ${ }^{1}$ In our case, treatments involving high temperatures cannot be however used due to the limited thermal stability of the polymeric resins, ${ }^{34}$ as shown in Figure 4. For resins, the treatment used for varying the metal dispersion is based on the mobility of the reduced metal phase in aqueous media, in which a migration of $\mathrm{Pt}$ clusters occurs inside the polymer, forming bigger metal agglomerations. The accessibility of the resulting platinum particles should be improved if the active metal phase becomes concentrated on the outer layers of the polymer particles.

Figure 7 shows EP total conversion values as a function of time for 2Pt-WA30- $\Phi$ samples (with $\Phi=6,12,24,30$ and $48 \mathrm{~h}$, sintering time). Values of total conversion of $\mathrm{EP}$, initial reaction rate $\left(\mathrm{r}^{\circ}\right)$ and $\mathrm{ee}_{(\mathrm{R})}$ of fresh and sintered catalysts are shown in Table 3. The diminution of the $\mathrm{r}^{\circ}$ with $\Phi$ was attributed to the loss of metal dispersion. The ee ${ }_{(\mathbb{R})}$ results indicate that there is an optimal sintering degree, corresponding in this case to a sintering treatment of $24 \mathrm{~h}$. A longer sintering of $48 \mathrm{~h}$ fails to produce catalysts with higher $\mathrm{ee}_{(\mathrm{R})}$. The optimal degree of sintering should correspond to an optimal Pt particle size in these conditions.

Pt crystals with no interaction with the support should develop particles with morphologies dictated only by

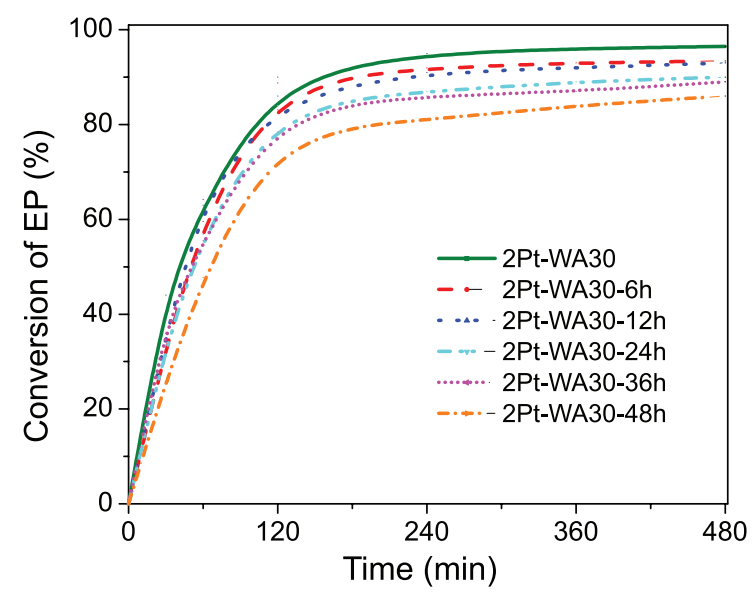

Figure 7. Activity of fresh and sintered Pt catalysts $\left(\mathrm{W}_{\text {cat }}=0.25 \mathrm{~g}\right.$, $\mathrm{V}_{\text {solvent }}=50 \mathrm{~mL},[\mathrm{EP}]=0.176 \mathrm{M},[\mathrm{CD}]=0.02 \mathrm{~g} \mathrm{~L}^{-1}, \mathrm{~T}=298 \mathrm{~K}$, $\mathrm{p}_{\mathrm{H} 2}=40$ bar $)$.

thermodynamic effects. In order to minimize their surface energy, mostly planes with low surface energy should be found. For fully centered cubic Pt particles, these planes would be the $<111>\left(2.299 \mathrm{~J} \mathrm{~m}^{-2}\right)$, the $<100>\left(2.734 \mathrm{~J} \mathrm{~m}^{-2}\right)$ and the $<110\rangle\left(2.819 \mathrm{~J} \mathrm{~m}^{-2}\right) .{ }^{35}$ Atoms on plane faces will have coordination number $(\mathrm{CN})$ of $8-9$, atoms on edges $\mathrm{CN}=7$ and atoms on corners $\mathrm{CN}=6$. Due to the higher energy of atoms of low coordination, a spherical particle is not likely, but a polyhedron resembling it. For $\mathrm{Pt}$, this is the cube-octahedral particle, with $<111>(\mathrm{CN}=9)$ and $<100\rangle$ $(\mathrm{CN}=8)$ as main faces. For such a Pt cubo-octahedral particle, some geometrical relations can be calculated in comparison to the size of a cinchonidine molecule. The quinoline group of cinchonidine has a size of about $0.6 \mathrm{~nm}$. Some authors indicate that adsorption of cinchonidine on regular Pt faces could occur mainly by adsorption of the

Table 3. Effect of chiral modifier (CM) nature, sintering, swelling and reuse, on the activity and enantioselectivity of Pt catalysts

\begin{tabular}{|c|c|c|c|c|c|}
\hline Catalyst & Condition $^{\mathrm{a}}$ & $\mathrm{d}_{\mathrm{TEM}} / \mathrm{nm}$ & $\begin{array}{c}\text { Conversion } / \% \\
t=60 \mathrm{~min}\end{array}$ & $\begin{array}{c}\text { ee } / \% \\
t=60 \text { min }\end{array}$ & $\begin{array}{c}\mathrm{r}^{\circ} \times 10^{2} / \\
\left(\mathrm{mol} \mathrm{g} \mathrm{ge}_{\mathrm{Me}} \min ^{-1}\right)\end{array}$ \\
\hline 2Pt-WA30 & b & $1.2-5.0$ & 14.33 & 0 & 0.422 \\
\hline 2Pt-WA30 & \multirow{6}{*}{$\mathrm{c}$} & $1.2-5.0$ & 63.01 & (R) 18.15 & 1.988 \\
\hline 2Pt-WA30-6h & & $1.2-8.0$ & 59.56 & (R) 20.73 & 1.795 \\
\hline 2Pt-WA30-12h & & $1.2-9.0$ & 58.50 & (R) 23.18 & 1.766 \\
\hline 2Pt-WA30-24h & & \multirow{2}{*}{$1.2-10.0$} & 56.84 & (R) 27.55 & 1.724 \\
\hline Reuse 1 & & & 54.70 & (R) 25.63 & 1.701 \\
\hline 2Pt-WA30-36h & & $1.2-11.0$ & 55.50 & (R) 22.67 & 1.625 \\
\hline 2Pt-WA30-48h & & $1.2-11.0$ & 47.79 & (R) 22.61 & 1.425 \\
\hline 2Pt-WA30 & d & $1.2-5.0$ & 43.87 & (S) 13.08 & 1.126 \\
\hline 2Pt-WA30-SW & \multirow{3}{*}{ e } & \multirow{3}{*}{$1.2-5.0$} & 36.75 & (R) 23.08 & 0.936 \\
\hline Reuse 1 & & & 21.86 & (R) 21.75 & 0.672 \\
\hline Reuse 2 & & & 20.57 & (R) 11.51 & 0.601 \\
\hline
\end{tabular}

$\mathrm{d}_{\text {TEM }}$ : metal particle diameter size; $t$ : time; ee: enantioselectivity; $\mathrm{r}_{\mathrm{o}}$ : initial reaction rate; ${ }^{\text {in }}$ all cases: $\mathrm{W}_{\text {cat }}=0.25 \mathrm{~g}, \mathrm{~V}_{\text {solvent }}=50 \mathrm{~mL}, \mathrm{~T}=298 \mathrm{~K}, \mathrm{p}=40 \mathrm{bar}$ and $[\mathrm{PE}]=0.176 \mathrm{M}$; ${ }^{\mathrm{b}}$ no $\mathrm{CM}$ was used; $\mathrm{c}[\mathrm{CD}]=0.02 \mathrm{~g} \mathrm{~L}^{-1} ;{ }^{\mathrm{d}}[\mathrm{CN}]=0.0078 \mathrm{~g} \mathrm{~L}^{-1}$; ${ }^{\mathrm{e}}$ no extra $\mathrm{CM}$ was added to the reaction media. 
quinoline group on the $\mathrm{Pt}<111>$ face in a flat fashion, occupying a cluster of $7 \mathrm{Pt}$ atoms of hexagonal shape. ${ }^{36}$ The rest of the molecule would not be in contact with the surface.

The enantioselectivity of the different time sintered samples could be associated to the density of Pt particles that have a size that allows CD adsorption. In this sense, cinchonidine can be estimated to occupy about $19 \mathrm{Pt}$ atoms. A Pt particle of $50 \%$ dispersion has a size (diameter) of about $2.5 \mathrm{~nm}$ and about 230 surface atoms. For this particle, about $80 \%$ are $<111>$ atoms distributed on 8 faces. This means that a particle of about $50 \%$ dispersion accommodates 1 cinchonidine molecule per $<111>$ face and 8 molecules in total. This should be the particle size with probably the best catalytic effect because: $(i)$ smaller particles can have a lower affinity for the quinoline group; (ii) bigger particles can accommodate 2 quinoline groups only if the size is equal or higher than $5 \mathrm{~nm}$; (iii) the edgescinchonidine-reactant interaction is maximum.

The influence of the nature of the chiral modifier was also screened in Table 3. The use of (+)-cinchonine as $\mathrm{CM}$ leads to the formation of the opposite optically active compound, $(S)$-EL. The ee ${ }_{(S)}$ achieved value is smaller than the ee $e_{(\mathrm{R})}$ obtained with $\mathrm{CD}$. This selectivity pattern was confirmed by other reports. ${ }^{37-39}$

The effect of the swelling technique used for catalyst surface modification with CD can be seen in Table 3. A comparison was made of the results obtained using the conventional technique of Orito et al. ${ }^{5}$ and those obtained using the swelling technique. The same amount of chiral inducer was used. In the conventional technique, $\mathrm{CD}$ was added to the reaction media. In the swelling technique, CD was dissolved in methanol in order to let it diffuse into the porous structure during the pretreatment. For the swollen catalyst, no extra $\mathrm{CD}$ was further added to the reaction. The values of $\mathrm{ee}_{(\mathrm{R})}$ obtained with the 2Pt-WA30-SW catalyst were $27 \%$ higher than those obtained with the conventional procedure, suggesting a distribution of $\mathrm{CM}$ on the bulk Pt sites.

The reuse tests for the sintered and swollen catalyst show that the enantio differentiation remains at constant values for two cycles and then decreases. However, the activity of the catalyst decreases slightly with the number of cycles, note that no CD was added during the reusing tests of swollen catalyst. Moreover, the reuse of the catalysts prepared by the classical method, adding CD in each test, seems to be more robust in terms of activity and enantioselectivity, as it can be seen in Table 3. Reused swollen catalysts had a poorer performance and this was attributed to the occurrence of hydrogenation of $\mathrm{CM}$, thus affecting the metal-CM interaction.

\section{Conclusions}

Enantioselective hydrogenation of EP over noble metals supported on ionic exchange resins was demonstrated to be feasible. Of the four noble metals catalysts modified with $(-)$-cinchonidine, the decreasing order of selectivity to $(R)$-ethyl lactate was: $\mathrm{Pt}>>\mathrm{Pd}>\mathrm{Au}>$ $\mathrm{Rh}$, while the activity order was: $\mathrm{Rh}>>\mathrm{Pt}>\mathrm{Pd}>\mathrm{Au}$. The increment of Pt loading from 1 to $2 \%$ slightly improved the enantiodifferentiation. Conversely the use of (+)-cinchonine as chiral inducer produced a preferential formation of $(S)$-EL. The performance of the catalysts can be interpreted in terms of electronic effects between the metallic sites $\left(\mathrm{Me}^{\delta+}\right)$ with the substrate and $\mathrm{CM}$, hydrogen cleaving on filled d metal orbital $\left(\mathrm{Me}^{\circ}\right.$ sites), sorption strength and average particle diameter.

It was demonstrated that enantioselective hydrogenation of ethyl pyruvate is a structure-sensitive reaction, and that for a given amount of Pt loading, there exists a competing effect between the decrease of metal surface area due to particle sintering (leading to an overall yield reduction) and the improvement of the selectivity to the $(R)$-enantiomer when (-)-cinchonidine was used as chiral modifier.

A novel method for sintering Pt metal particles by nitriles reduction was used, observing a controlled migration of the metal particles to the surface layers. An optimal time of reduction treatment was found at $24 \mathrm{~h}$ that corresponds to a maximum enantiomeric excess of the sintered catalysts of $28 \%$. This optimal point should also correspond to an optimal Pt particle size in the range $1.2-10.0 \mathrm{~nm}$.

Modification of the catalyst surface using a swelling technique of $\mathrm{CM}$ was tried. The swollen catalyst showed an enantioselectivity (27\%) higher than the catalyst modified with Orito's technique, ${ }^{5}$ suggesting a better interaction between the CM and the bulk Pt sites.

The reuse of the catalysts was studied. The catalysts prepared by the classical method were more reusable. Reused swollen catalysts had a poorer performance and this was attributed to the occurrence of hydrogenation of $\mathrm{CM}$, thus affecting the metal-CM interaction.

\section{Acknowledgments}

We thank CONICET, ANPCyT and UNL for the financial assistance of this work (grants PIP 112-20110100410 and 112-201301-00457, PICT and CAI+D).

\section{References}

1. Baiker, A.; J. Mol. Catal. A: Chem. 1997, 115, 473. 
2. Noyori, R.; Angew. Chem., Int. Ed. 2002, 41, 2008.

3. Blaser, H.-U.; Studer, M ; Chirality 1999, 11, 459.

4. Studer, M.; Blaser, H.-U.; Exner, C.; Adv. Synth. Catal. 2003, $345,45$.

5. Niwa, S.; Imai, S.; Orito, Y.; Bull. Chem. Soc. Jpn. 1982, 1, 137.

6. Zuo, X.; Liu, H.; Tetrahedron 1999, 55, 7787.

7. Wehrli, J. T.; Baker, A.; Monti, D. M.; Blaser, H.-U.; J. Mol. Catal. 1990, 61, 207.

8. Attard, G.; Griffin, K.; Jenkins, D.; Johnston, P.; Wells, P.; Catal. Today 2006, 114, 346.

9. Barbaro, P.; Chem. Eur. J. 2006, 12, 5666.

10. Králik, M.; Biffis, A.; J. Mol. Catal. A: Chem. 2001, 177, 113.

11. Mendow, G.; Sánchez, A.; Grosso, C.; Querini, C. A; J. Environ. Chem. Eng. 2017, 5, 1404.

12. Mendow, G.; Marchesini, F. A.; Miró, E. E.; Querini, C. A.; Angew. Chem., Int. Ed. 2011, 50, 1911.

13. Carrara, N.; Badano, J. M.; Coloma-Pascual, F.; Vera, C.; Quiroga, M.; Chem. Pap. 2017, 71, 1669.

14. Carrara, N.; Badano, J. M.; Bertero, N.; Torres, G.; Betti, C.; Martínez-Bovier, L.; Quiroga, M.; Vera, C.; J. Chem. Technol. Biotechnol. 2014, 89, 265.

15. Carrara, N.; Badano, J. M.; Betti, C.; Lederhos, C.; Rintoul, I.; Coloma-Pascual, F.; Vera, C.; Quiroga, M.; Catal. Commun. 2015, 61, 72.

16. Wehrli, J. T.; Baiker, A.; Monti, D. M.; Blaser, H. U.; Jalett, H. P.; J. Mol. Catal. 1989, 57, 245.

17. Burgi, T.; Baiker, A.; J. Am. Soc. 1998, 12920.

18. Díaz-Auñon, J. A.; García-Martinez, J.; Román-Martinez, M. C.; Salinas-Martínez de Lecea, C.; Catal. Lett. 2001, 76, 41.

19. Petla, R. K.; Vivekanandhan, S.; Misra, M.; Mohanty, A. K.; Satyanarayana, N.; J. Biomater. Nanobiotechnol. 2012, 3, 14.

20. Marot, L.; De Temmerman, G.; Thommen, V.; Mathys, D.; Oelhafen, P.; Surf. Coat. Technol. 2008, 202, 2837.

21. Krishnamurthy, S.; Esterle, A.; Sharma, N. C.; Sahi, S. V.; Nanoscale Res. Lett. 2014, 9, 627.

22. Zhang, J.; Wang, X.; Wu, C.; Wang, H.; Yi, B.; React. Kinet. Catal. Lett. 2004, 83, 229.
23. Herrera-Méndez, H. D.; Roquero, P.; Smit, M. A.; Ordóñez, L. C.; Int. J. Electrochem. Sci. 2011, 6, 4454.

24. Scardf, P.; Trento, U.; J. Mater. Res. 1993, 8, 1829.

25. Andrzejewska, E.; Kusch, P.; Andrzejewski, M.; Polym. Degrad. Stab. 1993, 40, 27.

26. Badano, J. M.; Quiroga, M.; Betti, C.; Vera, C.; Canavese, S.; Coloma-Pascual, F.; Catal. Lett. 2010, 137, 35.

27. Gelbard, G.; Ind. Eng. Chem. Res. 2005, 44, 8468.

28. National Institute of Standarts and Technology (NIST); NIST $X$-ray Photoelectron Spectroscopy Database, http://srdata.nist. gov/xps, accessed in April 2019.

29. Burgi, T.; Baiker, A.; Acc. Chem. Res. 2004, 37, 909.

30. Minder, B.; Schiirch, M.; Mallat, T.; Baiker, A.; Catal. Lett. 1995, 31, 143.

31. Shriver, D.; Atkins, P.; Langford, C.; Inorganic Chemistry, $3^{\text {rd }}$ ed.; WH Freeman and Co.: New York, USA, 1994.

32. Blaser, H. U.; Jalett, H. P.; Monti, D. M.; Reber, J. F.; Wehrli, J. T.; Stud. Surf. Sci. Catal. 1988, 153.

33. Blaser, H.; Jalett, H.; Garland, M.; Studer, M.; Thies, H.; WirthTijani, A.; Catalyst 1998, 294, 282.

34. Wehrli, J. T.; Baiker, A.; Monti, D. M.; Blaser, H. U.; J. Mol. Catal. 1989, 49, 195.

35. Vitos, L.; Ruban, A. V; Skriver, H. L.; Kollar, J.; Surf. Sci. 1998, $411,186$.

36. Behzadi, B.; Vargas, A.; Ferri, D.; Ernst, K.; Baiker, A.; J. Phys. Chem. B 2006, 110, 17082.

37. Blaser, H. U.; Jalett, H. P.; Heterog. Catal. Fine Chem. 1993, $3,139$.

38. Blaser, H. U.; Jalett, H. P.; Monti, D. M.; Reber, J. F.; Wehrly, J. T.; Heterog. Catal. Fine Chem. 1988, 153.

39. Blaser, H. U.; Jalett, H. P.; Lottenbach, W.; Studer, M.; J. Am. Chem. Soc. 2000, 122, 12675.

Submitted: January 31, 2019

Published online: April 23, 2019 